\title{
Exact Solutions for Some Fractional Differential Equations
}

\author{
Abdullah Sonmezoglu \\ Department of Mathematics, Faculty of Science and Arts, Bozok University, 66100 Yozgat, Turkey \\ Correspondence should be addressed to Abdullah Sonmezoglu; abdullah.sonmezoglu@bozok.edu.tr
}

Received 10 February 2015; Accepted 29 April 2015

Academic Editor: Andrei D. Mironov

Copyright (C) 2015 Abdullah Sonmezoglu. This is an open access article distributed under the Creative Commons Attribution License, which permits unrestricted use, distribution, and reproduction in any medium, provided the original work is properly cited.

The extended Jacobi elliptic function expansion method is used for solving fractional differential equations in the sense of Jumarie's modified Riemann-Liouville derivative. By means of this approach, a few fractional differential equations are successfully solved. As a result, some new Jacobi elliptic function solutions including solitary wave solutions and trigonometric function solutions are established. The proposed method can also be applied to other fractional differential equations.

\section{Introduction}

Fractional differential equations attracted attention in physics, biology, engineering, signal processing, systems identification, control theory, finance, and fractional dynamics [13]. Also, they are employed in social sciences such as food supplement, climate, finance, and economics.

Finding approximate and exact solutions to fractional differential equations is an important task. Various analytical and numerical methods have been introduced to obtain solutions of fractional differential equations, such as the Adomian decomposition method $[4,5]$, the variational iteration method [6-8], the homotopy analysis method [9-12], the homotopy perturbation method [13-15], the Lagrange characteristic method [16], the finite difference method [17], the finite element method [18], the differential transformation method [19], the fractional subequation method [20-24], the first integral method [25], the $\left(G^{\prime} / G\right)$-expansion method [2629], the fractional complex transform method [30], and the modified simple equation method [31-33].

In [34], Jumarie proposed a modified Riemann-Liouville derivative. With this kind of fractional derivative and some useful formulas, we can convert fractional differential equations into integer-order differential equations by variable transformation.
In this paper, we used extended Jacobi elliptic function expansion method [35-37] to establish exact solutions for three nonlinear space-time fractional differential equations in the sense of Jumarie's modified RiemannLiouville derivative, namely, the space-time fractional generalized reaction duffing equation, the space-time fractional bidirectional wave equations, and the space-time fractional symmetric regularized long wave (SRLW) equation. Also, we included figures to show the properties of some Jacobi elliptic function solutions of these fractional differential equations.

\section{Jumarie's Modified Riemann-Liouville Derivative and the Extended Jacobi Elliptic Function Expansion Method}

In this section, we first give the definition and some properties of the modified Riemann-Liouville derivative which are used further in this paper.

The Jumarie modified Riemann-Liouville derivative of order $\alpha$ is defined by the expression [34] 


$$
D_{x}^{\alpha} f(x)= \begin{cases}\frac{1}{\Gamma(-\alpha)} \int_{0}^{x}(x-\xi)^{-\alpha-1}[f(\xi)-f(0)] d \xi, & \alpha<0, \\ \frac{1}{\Gamma(1-\alpha)} \frac{d}{d x} \int_{0}^{x}(x-\xi)^{-\alpha}[f(\xi)-f(0)] d \xi, & 0<\alpha<1, \\ {\left[f^{(n)}(x)\right]^{(\alpha-n)},} & n \leq \alpha<n+1, n \geq 1,\end{cases}
$$

where $f: R \rightarrow R, x \rightarrow f(x)$ denote a continuous (but not necessarily differentiable) function.

Some properties of the fractional modified RiemannLiouville derivative were summarized and three useful formulas of them are [34]

$$
\begin{aligned}
D_{x}^{\alpha} x^{r} & =\frac{\Gamma(1+r)}{\Gamma(1+r-\alpha)} x^{r-\alpha}, \\
D_{x}^{\alpha}(f(x) g(x)) & =g(x) D_{x}^{\alpha} f(x)+f(x) D_{x}^{\alpha} g(x), \\
D_{x}^{\alpha} f[g(x)] & =f_{g}^{\prime}[g(x)] D_{x}^{\alpha} g(x) \\
& =D_{g}^{\alpha} f[g(x)]\left(g^{\prime}(x)\right)^{\alpha} .
\end{aligned}
$$

Next, let us consider nonlinear partial fractional differential equation

$$
\begin{gathered}
P\left(u, D_{t}^{\alpha} u, D_{x}^{\beta} u, D_{y}^{\gamma} u, D_{t}^{\alpha} D_{t}^{\alpha} u, D_{t}^{\alpha} D_{x}^{\beta} u, D_{x}^{\beta} D_{x}^{\beta} u,\right. \\
\left.D_{x}^{\beta} D_{y}^{\gamma} u, D_{y}^{\gamma} D_{y}^{\gamma} u, \ldots\right)=0, \quad 0<\alpha, \beta, \gamma \leq 1,
\end{gathered}
$$

where $u$ is an unknown function and $P$ is a polynomial of $u$. In this equation, the partial fractional derivatives involving the highest order derivatives and the nonlinear terms are included.

$\mathrm{Li}$ and $\mathrm{He}$ [38] presented a fractional complex transform to convert fractional differential equations into ordinary differential equations (ODEs), so all analytical methods devoted to the advanced calculus can be easily applied to the fractional calculus. By using the traveling wave variable

$$
\begin{aligned}
u(x, y, t) & =U(\xi), \\
\xi & =\frac{\delta x^{\beta}}{\Gamma(1+\beta)}+\frac{\zeta y^{\gamma}}{\Gamma(1+\gamma)}+\frac{\lambda t^{\alpha}}{\Gamma(1+\alpha)},
\end{aligned}
$$

where $\delta, \zeta$ are nonzero arbitrary constants and $\lambda$ is the wave speed, we can rewrite (3) as the following nonlinear ODE:

$$
Q\left(U, U^{\prime}, U^{\prime \prime}, U^{\prime \prime \prime}, \ldots\right)=0
$$

where the prime denotes the derivation with respect to $\xi$. If possible, we should integrate (5) term by term one or more times.

Our main goal is to derive exact or at least approximate solutions, if possible, for this ODE. For this purpose, using the extended Jacobi elliptic function expansion method, $U(\xi)$ can be expressed as a finite series of Jacobi elliptic functions, sn $\xi$, that is, the ansatz:

$$
u(x, y, t)=U(\xi)=\sum_{j=0}^{n} a_{j} \mathrm{sn}^{j} \xi+\sum_{j=1}^{n} b_{j} \mathrm{sn}^{-j} \xi .
$$

The parameter $n$ is determined by balancing the linear term(s) of highest order with the nonlinear one(s). And

$$
\begin{aligned}
\mathrm{cn}^{2} \xi & =1-\operatorname{sn}^{2} \xi, \\
\operatorname{dn}^{2} \xi & =1-m^{2} \operatorname{sn}^{2} \xi, \\
\frac{d}{d \xi} \operatorname{sn} \xi & =\operatorname{cn} \xi \operatorname{dn} \xi \\
\frac{d}{d \xi} \operatorname{cn} \xi & =-\operatorname{sn} \xi \operatorname{dn} \xi \\
\frac{d}{d \xi} \operatorname{dn} \xi & =-m^{2} \operatorname{sn} \xi \operatorname{cn} \xi
\end{aligned}
$$

where $\mathrm{cn} \xi$ and $\mathrm{dn} \xi$ are the Jacobi elliptic cosine function and the Jacobi elliptic function of the third kind, respectively, with the modulus $m(0<m<1)$. Therefore, the highest degree of $d^{p} U / d \xi^{p}$ is taken as

$$
\begin{aligned}
O\left(\frac{d^{p} U}{d \xi^{p}}\right) & =n+p, \quad p=1,2,3, \ldots, \\
O\left(U^{q} \frac{d^{p} U}{d \xi^{p}}\right) & =(q+1) n+p, \\
q & =0,1,2, \ldots, p=1,2,3, \ldots .
\end{aligned}
$$

Substituting (6)-(8) into (5) and comparing the coefficients of each power of $\operatorname{sn} \xi$ in both sides, we get an overdetermined system of nonlinear algebraic equations with respect to $\lambda$, $a_{j}(j=0,1, \ldots, n)$, and $b_{j}(j=1,2, \ldots, n)$. Solving this system, with the aid of Mathematica, then $\lambda, a_{j}(j=$ $0,1, \ldots, n)$, and $b_{j}(j=1,2, \ldots, n)$ can be determined. Substituting these results into (6), then some new Jacobi elliptic function solutions of (3) can be obtained. We can get other kinds of Jacobi doubly periodic wave solutions. 
Since

$$
\begin{aligned}
& \lim _{m \rightarrow 1} \operatorname{sn} \xi=\tanh \xi, \\
& \lim _{m \rightarrow 1} \operatorname{cn} \xi=\operatorname{sech} \xi, \\
& \lim _{m \rightarrow 1} \operatorname{dn} \xi=\operatorname{sech} \xi, \\
& \lim _{m \rightarrow 0} \operatorname{sn} \xi=\sin \xi, \\
& \lim _{m \rightarrow 0} \operatorname{cn} \xi=\cos \xi, \\
& \lim _{m \rightarrow 0} \operatorname{dn} \xi=1,
\end{aligned}
$$

$u$ degenerates, respectively, as the following form.

(1) Solitary wave solutions:

$$
u(x, y, t)=\sum_{j=0}^{n} a_{j} \tanh ^{j} \xi+\sum_{j=1}^{n} b_{j} \operatorname{coth}^{j} \xi
$$

(2) Triangular function formal solution:

$$
u(x, y, t)=\sum_{j=0}^{n} a_{j} \sin ^{j} \xi+\sum_{j=1}^{n} b_{j} \csc ^{j} \xi .
$$

\section{Applications of the Method}

In this section, we present three examples to demonstrate the effectiveness of our approach to solve nonlinear fractional partial differential equations.

\subsection{Space-Time Fractional Generalized Reaction Duffing Equa-} tion. We have applied the extended Jacobi elliptic function expansion method to construct the exact solutions of spacetime fractional generalized reaction duffing equation $[39,40]$ in the form

$$
\frac{\partial^{2 \alpha} u}{\partial t^{2 \alpha}}+p \frac{\partial^{2 \alpha} u}{\partial x^{2 \alpha}}+q u+r u^{2}+s u^{3}=0, \quad 0<\alpha<1,
$$

where $p, q, r$, and $s$ are all constants. Equation (12) reduces many well-known nonlinear fractional wave equations such as the following.

(i) Fractional Klein-Gordon equation:

$$
\frac{\partial^{2 \alpha} u}{\partial t^{2 \alpha}}-\frac{\partial^{2 \alpha} u}{\partial x^{2 \alpha}}-a u-b u^{3}=0, \quad t>0,0<\alpha<1 .
$$

(ii) Fractional Landau-Ginzburg-Higgs equation:

$$
\frac{\partial^{2 \alpha} u}{\partial t^{2 \alpha}}-\frac{\partial^{2 \alpha} u}{\partial x^{2 \alpha}}-m^{2} u+g^{2} u^{3}=0, \quad t>0,0<\alpha<1 .
$$

(iii) Fractional $\varphi^{4}$ equation:

$$
\frac{\partial^{2 \alpha} u}{\partial t^{2 \alpha}}-\frac{\partial^{2 \alpha} u}{\partial x^{2 \alpha}}+u-u^{3}=0, \quad t>0,0<\alpha<1 .
$$

(iv) Fractional duffing equation:

$$
\frac{\partial^{2 \alpha} u}{\partial t^{2 \alpha}}+a u+b u^{3}=0, \quad t>0,0<\alpha<1
$$

(v) Fractional Sine-Gordon equation:

$$
\frac{\partial^{2 \alpha} u}{\partial t^{2 \alpha}}-\frac{\partial^{2 \alpha} u}{\partial x^{2 \alpha}}+u-\frac{1}{6} u^{3}=0, \quad t>0,0<\alpha<1 .
$$

For our purpose, we introduce the following transformations:

$$
\begin{aligned}
u(x, t) & =U(\xi), \\
\xi & =\frac{l x^{\alpha}}{\Gamma(1+\alpha)}-\frac{\lambda t^{\alpha}}{\Gamma(1+\alpha)},
\end{aligned}
$$

where $\xi$ is a wave variable and $l$ and $\lambda$ are constants; all of them are to be determined. Substituting (18) into (12), (12) is reduced into an ODE:

$$
\begin{gathered}
U^{\prime \prime}(\xi)+\frac{q}{\lambda^{2}+p l^{2}} U(\xi)+\frac{r}{\lambda^{2}+p l^{2}} U^{2}(\xi) \\
+\frac{s}{\lambda^{2}+p l^{2}} U^{3}(\xi)=0,
\end{gathered}
$$

where $U^{\prime}=d U / d \xi$. Suppose that the solution of (19) can be expressed by

$$
U(\xi)=\sum_{j=0}^{n} a_{j} \operatorname{sn}^{j} \xi+\sum_{j=1}^{n} b_{j} \mathrm{sn}^{-j} \xi
$$

Considering the homogeneous balance between the highest order derivative $U^{\prime \prime}$ and the highest order nonlinear term $U^{3}$ in (19), we obtain $n=1$. So

$$
U(\xi)=a_{0}+a_{1} \operatorname{sn} \xi+b_{1} \mathrm{sn}^{-1} \xi .
$$

Substituting (21) into (19) and comparing the coefficients of each power of $\operatorname{sn} \xi$ in both sides, we get an overdetermined system of nonlinear algebraic equations with respect to $\lambda, a_{0}$, $a_{1}$, and $b_{1}$. Solving this system with Mathematica, we get the following results.

Case 1. Consider

$$
\begin{aligned}
& a_{0}=-\frac{r}{3 s}, \\
& a_{1}= \pm \frac{\sqrt{2} m r}{3 \sqrt{s^{2}\left(1+m^{2}\right)}}, \\
& b_{1}=0, \\
& \lambda= \pm i \frac{\sqrt{r^{2}+9 l^{2} p s\left(1+m^{2}\right)}}{3 \sqrt{s\left(1+m^{2}\right)}}, \\
& q=\frac{2 r^{2}}{9 s} .
\end{aligned}
$$


Case 2. Consider

$$
\begin{aligned}
& a_{0}=-\frac{r}{3 s}, \\
& a_{1}=0, \\
& b_{1}= \pm \frac{\sqrt{2} r}{3 \sqrt{s^{2}\left(1+m^{2}\right)}}, \\
& \lambda= \pm i \frac{\sqrt{r^{2}+9 l^{2} p s\left(1+m^{2}\right)}}{3 \sqrt{s\left(1+m^{2}\right)}}, \\
& q=\frac{2 r^{2}}{9 s} .
\end{aligned}
$$

Case 3. Consider

$$
\begin{aligned}
& a_{0}=-\frac{r}{3 s}, \\
& a_{1}= \pm \frac{\sqrt{2} m r}{3 s \sqrt{1+m(6+m)}}, \\
& b_{1}= \pm \frac{\sqrt{2} r}{3 s \sqrt{1+m(6+m)}}, \\
& \lambda=-i \frac{\sqrt{r^{2}+9 l^{2} p s(1+m(6+m))}}{3 \sqrt{s(1+m(6+m))}}, \\
& q=\frac{2 r^{2}}{9 s} .
\end{aligned}
$$

Thus, we obtain the following solutions of (12).

Solution 1. See Figure 1:

$$
\begin{aligned}
u_{1} & =-\frac{r}{3 s} \pm \frac{\sqrt{2} m r}{3 \sqrt{s^{2}\left(1+m^{2}\right)}} \\
& \cdot \operatorname{sn}\left(\frac{l x^{\alpha}}{\Gamma(1+\alpha)} \mp i \frac{\sqrt{r^{2}+9 l^{2} p s\left(1+m^{2}\right)}}{3 \Gamma(1+\alpha) \sqrt{s\left(1+m^{2}\right)}} t^{\alpha}\right) .
\end{aligned}
$$

Solution 2. See Figure 2:

$$
\begin{aligned}
& u_{2}=-\frac{r}{3 s} \pm \frac{\sqrt{2} r}{3 \sqrt{s^{2}\left(1+m^{2}\right)}} \\
& \cdot \operatorname{sn}^{-1}\left(\frac{l x^{\alpha}}{\Gamma(1+\alpha)} \mp i \frac{\sqrt{r^{2}+9 l^{2} p s\left(1+m^{2}\right)}}{3 \Gamma(1+\alpha) \sqrt{s\left(1+m^{2}\right)}} t^{\alpha}\right) .
\end{aligned}
$$

Solution 3. Consider

$$
\begin{aligned}
u_{3} & =-\frac{r}{3 s} \pm \frac{\sqrt{2} r}{3 s \sqrt{1+m(6+m)}} \times\left[m s n \left(\frac{l x^{\alpha}}{\Gamma(1+\alpha)}\right.\right. \\
& \left.+i \frac{\sqrt{r^{2}+9 l^{2} p s(1+m(6+m))}}{3 \Gamma(1+\alpha) \sqrt{s(1+m(6+m))}} t^{\alpha}\right) \\
& +\operatorname{sn}^{-1}\left(\frac{l x^{\alpha}}{\Gamma(1+\alpha)}\right. \\
& \left.\left.+i \frac{\sqrt{r^{2}+9 l^{2} p s(1+m(6+m))}}{3 \Gamma(1+\alpha) \sqrt{s(1+m(6+m))}} t^{\alpha}\right)\right]
\end{aligned}
$$

3.1.1. Soliton Solutions. When the modulus $m$ approaches to 1 in (25), (26), and (27), we can obtain solitary wave solutions of space-time fractional generalized reaction duffing equation, respectively:

$$
\begin{aligned}
u_{4}= & \frac{r}{3 s} \\
& \pm \frac{r}{3 s} \tanh \left(\frac{l x^{\alpha}}{\Gamma(1+\alpha)} \mp i \frac{\sqrt{r^{2}+18 l^{2} p s}}{3 \sqrt{2 s} \Gamma(1+\alpha)} t^{\alpha}\right), \\
u_{5}= & \frac{r}{3 s} \\
& \pm \frac{r}{3 s} \operatorname{coth}\left(\frac{l x^{\alpha}}{\Gamma(1+\alpha)} \mp i \frac{\sqrt{r^{2}+18 l^{2} p s}}{3 \sqrt{2 s} \Gamma(1+\alpha)} t^{\alpha}\right), \\
u_{6}= & \left.\frac{r}{3 s}\right) \\
& \pm \frac{r}{3 s} \operatorname{coth} 2\left(\frac{l x^{\alpha}}{\Gamma(1+\alpha)}+i \frac{\sqrt{r^{2}+72 l^{2} p s}}{3 \sqrt{8 s} \Gamma(1+\alpha)} t^{\alpha}\right) .
\end{aligned}
$$

3.1.2. Triangular Periodic Solutions. When the modulus $m$ approaches to zero in (26), (27), we can obtain trigonometric function solutions of space-time fractional generalized reaction duffing equation, respectively:

$$
\begin{aligned}
u_{7}= & -\frac{r}{3 s} \\
& \pm \frac{\sqrt{2} r}{3 s} \csc \left(\frac{l x^{\alpha}}{\Gamma(1+\alpha)} \mp i \frac{\sqrt{r^{2}+9 l^{2} p s}}{3 \sqrt{s} \Gamma(1+\alpha)} t^{\alpha}\right),
\end{aligned}
$$




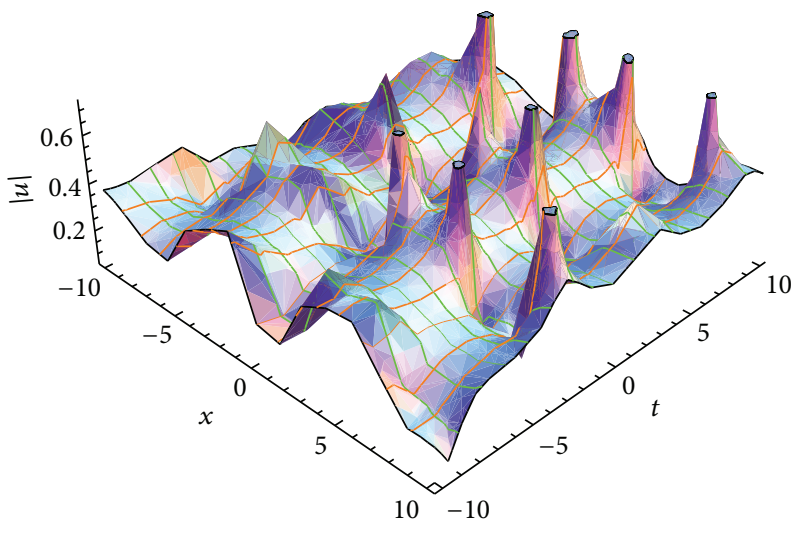

(a)



(b)

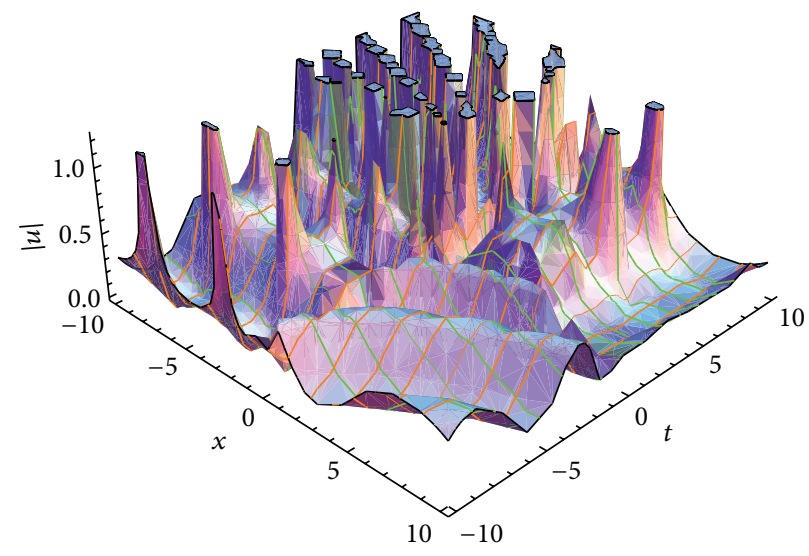

(c)

Figure 1: Profiles of $|u|$ in (25) corresponding to the values $m=0.1, \alpha=0.9, p=r=s=l=1 ; m=0.9, \alpha=0.2, p=r=s=l=1$; and $m=\alpha=0.5, p=l=3, r=s=-4$ from (a) to (c).

$$
\begin{aligned}
u_{8}= & -\frac{r}{3 s} \pm \frac{\sqrt{2} r}{3 s} \\
& +\csc \left(\frac{l x^{\alpha}}{\Gamma(1+\alpha)}+i \frac{\sqrt{r^{2}+9 l^{2} p s}}{3 \sqrt{s} \Gamma(1+\alpha)} t^{\alpha}\right) .
\end{aligned}
$$

3.2. Space-Time Fractional Bidirectional Wave Equations. Let us apply our method to the space-time fractional bidirectional wave equations in the form $[41,42]$

$$
\begin{aligned}
& D_{t}^{\alpha} v+D_{x}^{\alpha} u+u D_{x}^{\alpha} v+v D_{x}^{\alpha} u+a D_{x}^{\alpha} D_{x}^{\alpha} D_{x}^{\alpha} u \\
& -b D_{x}^{\alpha} D_{x}^{\alpha} D_{t}^{\alpha} v=0, \\
& D_{t}^{\alpha} u+D_{x}^{\alpha} v+u D_{x}^{\alpha} u+c D_{x}^{\alpha} D_{x}^{\alpha} D_{x}^{\alpha} v \\
& -d D_{x}^{\alpha} D_{x}^{\alpha} D_{t}^{\alpha} u=0,
\end{aligned}
$$

$$
0<\alpha \leq 1
$$

where $x$ represents the distance along the channel, $t$ is the elapsed time, the variable $u(x, t)$ is the dimensionless horizontal velocity, $v(x, t)$ is the dimensionless deviation of the water surface from its undisturbed position, and $a, b$, $c$, and $d$ are real constants. When $\alpha=1,(30)$ is the generalization of bidirectional wave equations, which can be used as a model equation for the propagation of long waves on the surface of water with a small amplitude by Bona and Chen [43].

For our purpose, we use the following transformation:

$$
\begin{aligned}
u(x, t) & =U(\xi), \\
v(x, t) & =V(\xi), \\
\xi & =\frac{R x^{\alpha}}{\Gamma(1+\alpha)}+\frac{S t^{\alpha}}{\Gamma(1+\alpha)},
\end{aligned}
$$

where $R$ and $S$ are nonzero constants. Substituting (31) into (30), we obtain

$$
\begin{aligned}
& S V^{\prime}+R U^{\prime}+R U V^{\prime}+R V U^{\prime}+a R^{3} U^{\prime \prime \prime}-b R^{2} S V^{\prime \prime \prime} \\
& \quad=0, \\
& S U^{\prime}+R V^{\prime}+R U U^{\prime}+c R^{3} V^{\prime \prime \prime}-d R^{2} S U^{\prime \prime \prime}=0,
\end{aligned}
$$






(a)

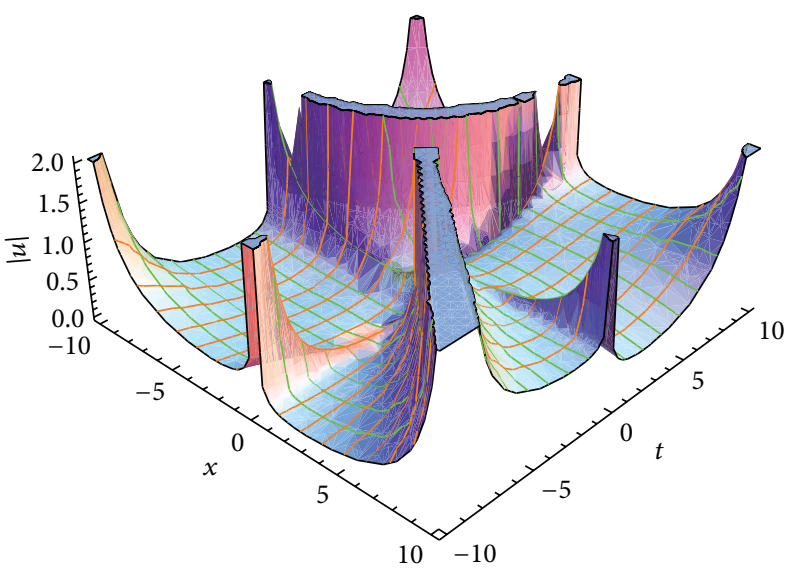

(b)

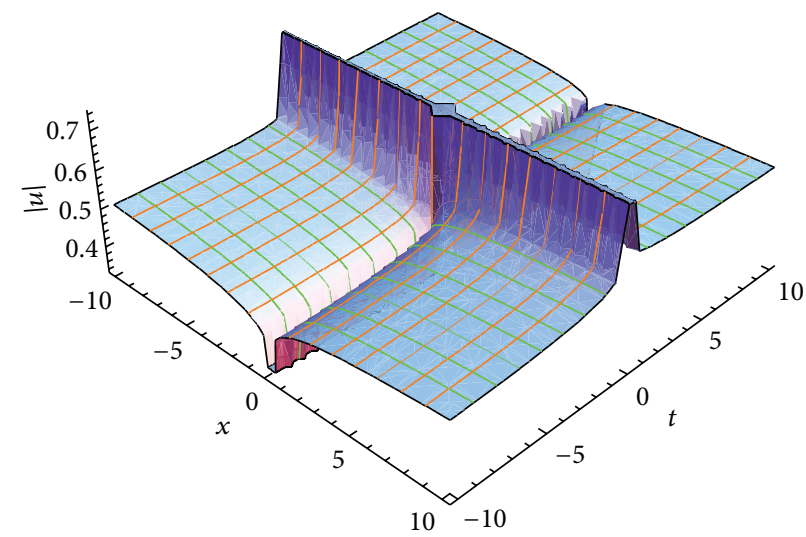

(c)

Figure 2: Profiles of $|u|$ in (26) corresponding to the values $m=0.5, \alpha=0.9, p=r=s=l=1 ; m=\alpha=0.5, p=r=s=l=1$; and $m=0.4$, $\alpha=0.1, l=1, p=r=s=2$ from (a) to (c).

where $U^{\prime}=d U / d \xi$. Suppose that the solutions of (32) can be expressed by

$$
\begin{array}{r}
U(\xi)=\sum_{j=0}^{n_{1}} a_{j} \mathrm{sn}^{j} \xi+\sum_{j=1}^{n_{1}} b_{j} \mathrm{sn}^{-j} \xi, \\
V(\xi)=\sum_{j=0}^{n_{2}} c_{j} \mathrm{sn}^{j} \xi+\sum_{j=1}^{n_{2}} d_{j} \mathrm{sn}^{-j} \xi .
\end{array}
$$

Balancing the highest order derivative terms and nonlinear terms in (32), we can obtain $n_{1}=n_{2}=2$. So we have

$$
\begin{aligned}
& U(\xi)=a_{0}+a_{1} \operatorname{sn} \xi+a_{2} \operatorname{sn}^{2} \xi+b_{1} \operatorname{sn}^{-1} \xi+b_{2} \operatorname{sn}^{-2} \xi \\
& V(\xi)=c_{0}+c_{1} \operatorname{sn} \xi+c_{2} \operatorname{sn}^{2} \xi+d_{1} \operatorname{sn}^{-1} \xi+d_{2} \operatorname{sn}^{-2} \xi
\end{aligned}
$$

Proceeding as in the previous case, we get the following results.
Case 1. Consider

$$
\begin{aligned}
& a_{1}=b_{1}=b_{2}=c_{1}=d_{1}=d_{2}=0, \\
& c_{2}=c_{2},
\end{aligned}
$$

$a_{0}$

$$
=i \frac{2(c+d)\left(c_{2}+6 a m^{2} R^{2}\right)-b c_{2}\left(1-4 c R^{2}\left(1+m^{2}\right)\right)}{2 R \sqrt{3 b c m^{2}\left(b c_{2}-2 d\left(c_{2}+6 a m^{2} R^{2}\right)\right)}},
$$

$a_{2}=-i \frac{2 \sqrt{3 c} b c_{2} m^{2} R}{\sqrt{b m^{2}\left(b c_{2}-2 d\left(c_{2}+6 a m^{2} R^{2}\right)\right)}}$

$c_{0}$

$$
=-\frac{b\left(c_{2}+4 c R^{2}\left(c_{2}+m^{2}\left(3+c_{2}\right)\right)\right)-2 d\left(c_{2}+6 a m^{2} R^{2}\right)}{12 b c m^{2} R^{2}},
$$

$S=-i \frac{\sqrt{c}\left(c_{2}+6 a m^{2} R^{2}\right)}{\sqrt{3 b m^{2}\left(b c_{2}-2 d\left(c_{2}+6 a m^{2} R^{2}\right)\right)}}$. 
Case 2. Consider

$$
\begin{aligned}
& a_{1}=b_{1}=c_{1}=d_{1}=0 \text {, } \\
& c_{2}=c_{2} \text {, } \\
& a_{0}=i \frac{2(c+d)\left(c_{2}+6 a m^{2} R^{2}\right)-b c_{2}\left(1-4 c R^{2}\left(1+m^{2}\right)\right)}{2 R \sqrt{3 b c m^{2}\left(b c_{2}-2 d\left(c_{2}+6 a m^{2} R^{2}\right)\right)}}, \\
& a_{2}=-i \frac{2 \sqrt{3 c} b c_{2} m^{2} R}{\sqrt{b m^{2}\left(b c_{2}-2 d\left(c_{2}+6 a m^{2} R^{2}\right)\right)}} \\
& c_{0} \\
& =-\frac{b\left(c_{2}+4 c R^{2}\left(c_{2}+m^{2}\left(3+c_{2}\right)\right)\right)-2 d\left(c_{2}+6 a m^{2} R^{2}\right)}{12 b c m^{2} R^{2}}, \\
& b_{2}=-i \frac{2 \sqrt{3 c} b c_{2} R}{\sqrt{b m^{2}\left(b c_{2}-2 d\left(c_{2}+6 a m^{2} R^{2}\right)\right)}} \\
& S=-i \frac{\sqrt{c}\left(c_{2}+6 a m^{2} R^{2}\right)}{\sqrt{3 b m^{2}\left(b c_{2}-2 d\left(c_{2}+6 a m^{2} R^{2}\right)\right)}}, \\
& d_{2}=\frac{c_{2}}{m^{2}} \text {. }
\end{aligned}
$$

Thus, we obtain the following solutions of (30).

Solution 1. Consider

$$
\begin{aligned}
u_{1} & =i \frac{2(c+d)\left(c_{2}+6 a m^{2} R^{2}\right)-b c_{2}\left(1-4 c R^{2}\left(1+m^{2}\right)\right)}{2 R \sqrt{3 b c m^{2}\left(b c_{2}-2 d\left(c_{2}+6 a m^{2} R^{2}\right)\right)}} \\
& -i \frac{2 \sqrt{3 c} b c_{2} m^{2} R}{\sqrt{b m^{2}\left(b c_{2}-2 d\left(c_{2}+6 a m^{2} R^{2}\right)\right)}} \\
& \cdot \operatorname{sn}^{2}\left(\frac{R x^{\alpha}}{\Gamma(1+\alpha)}+\frac{S}{\Gamma(1+\alpha)} t^{\alpha}\right), \\
v_{1} & =-\frac{b\left(c_{2}+4 c R^{2}\left(c_{2}+m^{2}\left(3+c_{2}\right)\right)\right)-2 d\left(c_{2}+6 a m^{2} R^{2}\right)}{12 b c m^{2} R^{2}} \\
& +c_{2} \operatorname{sn}^{2}\left(\frac{R x^{\alpha}}{\Gamma(1+\alpha)}+\frac{S}{\Gamma(1+\alpha)} t^{\alpha}\right) .
\end{aligned}
$$

Solution 2. Consider

$$
\begin{aligned}
u_{2} & =i \frac{2(c+d)\left(c_{2}+6 a m^{2} R^{2}\right)-b c_{2}\left(1-4 c R^{2}\left(1+m^{2}\right)\right)}{2 R \sqrt{3 b c m^{2}\left(b c_{2}-2 d\left(c_{2}+6 a m^{2} R^{2}\right)\right)}} \\
& -i \frac{2 \sqrt{3 c} b c_{2} R}{\sqrt{b m^{2}\left(b c_{2}-2 d\left(c_{2}+6 a m^{2} R^{2}\right)\right)}}\left[m ^ { 2 } \operatorname { s n } ^ { 2 } \left(\frac{R x^{\alpha}}{\Gamma(1+\alpha)}\right.\right. \\
& \left.\left.+\frac{S}{\Gamma(1+\alpha)} t^{\alpha}\right)+\operatorname{sn}^{-2}\left(\frac{R x^{\alpha}}{\Gamma(1+\alpha)}+\frac{S}{\Gamma(1+\alpha)} t^{\alpha}\right)\right]
\end{aligned}
$$

$$
\begin{aligned}
v_{2} & =-\frac{b\left(c_{2}+4 c R^{2}\left(c_{2}+m^{2}\left(3+c_{2}\right)\right)\right)-2 d\left(c_{2}+6 a m^{2} R^{2}\right)}{12 b c m^{2} R^{2}} \\
& +c_{2} \operatorname{sn}^{2}\left(\frac{R x^{\alpha}}{\Gamma(1+\alpha)}+\frac{S}{\Gamma(1+\alpha)} t^{\alpha}\right)+\frac{c_{2}}{m^{2}} \operatorname{sn}^{-2}\left(\frac{R x^{\alpha}}{\Gamma(1+\alpha)}\right. \\
& \left.+\frac{S}{\Gamma(1+\alpha)} t^{\alpha}\right),
\end{aligned}
$$

where

$$
S=-i \frac{\sqrt{c}\left(c_{2}+6 a m^{2} R^{2}\right)}{\sqrt{3 b m^{2}\left(b c_{2}-2 d\left(c_{2}+6 a m^{2} R^{2}\right)\right)}} .
$$

3.2.1. Soliton Solutions. When the modulus $m$ approaches to 1 in (37), (38), we can obtain solitary wave solutions of the space-time fractional bidirectional wave equations, respectively:

$$
\begin{aligned}
& u_{3}=i \frac{2(c+d)\left(c_{2}+6 a R^{2}\right)-b c_{2}\left(1-8 c R^{2}\right)}{2 R \sqrt{3 b c\left(b c_{2}-2 d\left(c_{2}+6 a R^{2}\right)\right)}}-i \\
& \frac{2 \sqrt{3 c} b c_{2} R}{\sqrt{b\left(b c_{2}-2 d\left(c_{2}+6 a R^{2}\right)\right)}} \\
& \cdot \tanh ^{2}\left(\frac{R x^{\alpha}}{\Gamma(1+\alpha)}+\frac{S}{\Gamma(1+\alpha)} t^{\alpha}\right) \\
& v_{3}=-\frac{b\left(c_{2}+4 c R^{2}\left(3+2 c_{2}\right)\right)-2 d\left(c_{2}+6 a R^{2}\right)}{12 b c R^{2}}+c_{2} \\
& \cdot \tanh ^{2}\left(\frac{R x^{\alpha}}{\Gamma(1+\alpha)}+\frac{S}{\Gamma(1+\alpha)} t^{\alpha}\right) \\
& u_{4}=i \frac{2(c+d)\left(c_{2}+6 a R^{2}\right)-b c_{2}\left(1-8 c R^{2}\right)}{2 R \sqrt{3 b c\left(b c_{2}-2 d\left(c_{2}+6 a R^{2}\right)\right)}}-i \\
& \frac{4 \sqrt{3 c} b c_{2} R}{\sqrt{b\left(b c_{2}-2 d\left(c_{2}+6 a R^{2}\right)\right)}}[1 \\
& \left.+2 \operatorname{csch}^{2} 2\left(\frac{R x^{\alpha}}{\Gamma(1+\alpha)}+\frac{S}{\Gamma(1+\alpha)} t^{\alpha}\right)\right] \text {, } \\
& v_{4}=-\frac{b\left(c_{2}+4 c R^{2}\left(3+2 c_{2}\right)\right)-2 d\left(c_{2}+6 a R^{2}\right)}{12 b c m^{2} R^{2}} \\
& +2 c_{2}\left[1+2 \operatorname{csch}^{2} 2\left(\frac{R x^{\alpha}}{\Gamma(1+\alpha)}+\frac{S}{\Gamma(1+\alpha)} t^{\alpha}\right)\right],
\end{aligned}
$$

where

$$
S=-i \frac{\sqrt{c}\left(c_{2}+6 a R^{2}\right)}{\sqrt{3 b\left(b c_{2}-2 d\left(c_{2}+6 a R^{2}\right)\right)}} .
$$


3.3. The Space-Time Nonlinear Fractional SRLW Equation. We consider the space-time nonlinear fractional SRLW equation $[44,45]$

$$
\begin{gathered}
D_{t}^{2 \alpha} u+D_{x}^{2 \alpha} u+u D_{t}^{\alpha}\left(D_{x}^{\alpha} u\right)+D_{t}^{\alpha} u D_{x}^{\alpha} u \\
+D_{t}^{2 \alpha}\left(D_{x}^{2 \alpha} u\right)=0, \quad 0<\alpha \leq 1,
\end{gathered}
$$

which arises in several physical applications including ion sound waves in plasma. For our purpose, we use the following transformation:

$$
\begin{aligned}
u(x, t) & =U(\xi), \\
\xi & =\frac{k x^{\alpha}}{\Gamma(1+\alpha)}+\frac{c t^{\alpha}}{\Gamma(1+\alpha)}+\xi_{0},
\end{aligned}
$$

where $k, c$, and $\xi_{0}$ are constants with $k, c \neq 0$. Substituting (43) into (42), we obtain

$$
2 k^{2} c^{2} U^{\prime \prime}+2\left(k^{2}+c^{2}\right) U+k c U^{2}=0 .
$$

Suppose that the solutions of (44) can be expressed by

$$
U(\xi)=\sum_{j=0}^{n} a_{j} \mathrm{sn}^{j} \xi+\sum_{j=1}^{n} b_{j} \mathrm{sn}^{-j} \xi .
$$

Considering the homogeneous balance between the highest order derivative $U^{\prime \prime}$ and the highest order nonlinear term $U^{2}$ in (44), we obtain $n=2$. So we have

$$
U(\xi)=a_{0}+a_{1} \operatorname{sn} \xi+a_{2} \operatorname{sn}^{2} \xi+b_{1} \operatorname{sn}^{-1} \xi+b_{2} \operatorname{sn}^{-2} \xi .
$$

Proceeding as in the previous cases, we get the following results.

Case 1. Consider

$$
\begin{aligned}
& a_{1}=b_{1}=b_{2}=0, \\
& a_{2}=-12 c k m^{2}, \\
& a_{0}=4 c k\left(1+m^{2}+\sqrt{1-m^{2}+m^{4}}\right),
\end{aligned}
$$

where

$$
c=i \sqrt{\frac{k^{2}}{1+4 k^{2} \sqrt{1-m^{2}+m^{4}}}} .
$$

Case 2. Consider

$$
\begin{aligned}
& a_{1}=a_{2}=b_{1}=0, \\
& b_{2}=-12 c k, \\
& a_{0}=4 c k\left(1+m^{2}-\sqrt{1-m^{2}+m^{4}}\right),
\end{aligned}
$$

where

$$
c=i \sqrt{\frac{k^{2}}{1-4 k^{2} \sqrt{1-m^{2}+m^{4}}}} .
$$

Case 3. Consider

$$
\begin{aligned}
& a_{1}=b_{1}=0, \\
& a_{2}=-12 c k m^{2}, \\
& b_{2}=-12 c k, \\
& a_{0}=4 c k\left(1+m^{2}+\sqrt{1+14 m^{2}+m^{4}}\right),
\end{aligned}
$$

where

$$
c=i \sqrt{\frac{k^{2}}{1+4 k^{2} \sqrt{1+14 m^{2}+m^{4}}}} .
$$

Thus, we obtain the following solutions of (42).

Solution 1. Consider

$$
\begin{aligned}
u_{1} & =4 c k\left(1+m^{2}+\sqrt{1-m^{2}+m^{4}}\right) \\
& -12 c k m^{2} \operatorname{sn}^{2}\left(\frac{k x^{\alpha}}{\Gamma(1+\alpha)}\right. \\
& \left.+\frac{i}{\Gamma(1+\alpha)} \sqrt{\frac{k^{2}}{1+4 k^{2} \sqrt{1-m^{2}+m^{4}}}} t^{\alpha}+\xi_{0}\right) .
\end{aligned}
$$

Solution 2. Consider

$$
\begin{aligned}
u_{2} & =4 c k\left(1+m^{2}-\sqrt{1-m^{2}+m^{4}}\right) \\
& -12 c k \mathrm{sn}^{-2}\left(\frac{k x^{\alpha}}{\Gamma(1+\alpha)}\right. \\
& \left.+\frac{i}{\Gamma(1+\alpha)} \sqrt{\frac{k^{2}}{1-4 k^{2} \sqrt{1-m^{2}+m^{4}}}} t^{\alpha}+\xi_{0}\right) .
\end{aligned}
$$

Solution 3. Consider

$$
\begin{aligned}
u_{3} & =4 c k\left(1+m^{2}+\sqrt{1+14 m^{2}+m^{4}}\right) \\
& -12 c k\left[m ^ { 2 } \operatorname { s n } ^ { 2 } \left(\frac{k x^{\alpha}}{\Gamma(1+\alpha)}\right.\right. \\
& \left.+\frac{i}{\Gamma(1+\alpha)} \sqrt{\frac{k^{2}}{1+4 k^{2} \sqrt{1+14 m^{2}+m^{4}}}} t^{\alpha}+\xi_{0}\right) \\
& +\operatorname{sn}^{-2}\left(\frac{k x^{\alpha}}{\Gamma(1+\alpha)}\right.
\end{aligned}
$$

$$
\left.\left.+\frac{i}{\Gamma(1+\alpha)} \sqrt{\frac{k^{2}}{1+4 k^{2} \sqrt{1+14 m^{2}+m^{4}}}} t^{\alpha}+\xi_{0}\right)\right] .
$$


3.3.1. Soliton Solutions. When the modulus $m$ approaches to 1 in (53), (54), and (55), we can obtain solitary wave solutions of the space-time nonlinear fractional SRLW equation, respectively:

$$
\begin{aligned}
u_{4} & =12 c k \operatorname{sech}^{2}\left(\frac{k x^{\alpha}}{\Gamma(1+\alpha)}+\frac{i}{\Gamma(1+\alpha)} \sqrt{\frac{k^{2}}{1+4 k^{2}}} t^{\alpha}\right. \\
& \left.+\xi_{0}\right) \\
u_{5} & =-8 c k-12 c k \operatorname{csch}^{2}\left(\frac{k x^{\alpha}}{\Gamma(1+\alpha)}\right. \\
& +\frac{i}{\Gamma(1+\alpha)} \sqrt{\left.\frac{k^{2}}{1-4 k^{2}} t^{\alpha}+\xi_{0}\right)} \\
u_{6} & =-48 c k \operatorname{csch}^{2} 2\left(\frac{k x^{\alpha}}{\Gamma(1+\alpha)}\right. \\
& \left.+\frac{i}{\Gamma(1+\alpha)} \sqrt{\frac{k^{2}}{1+16 k^{2}} t^{\alpha}+\xi_{0}}\right) .
\end{aligned}
$$

3.3.2. Triangular Periodic Solutions. We can obtain trigonometric function solutions of the space-time nonlinear fractional SRLW equation, when the modulus $m$ approaches to zero; for example, (54), (55) give the same solution:

$$
\begin{aligned}
& u_{7}=-12 c k \\
& \cdot \csc ^{2}\left(\frac{k x^{\alpha}}{\Gamma(1+\alpha)}+\frac{i}{\Gamma(1+\alpha)} \sqrt{\frac{k^{2}}{1-4 k^{2}}} t^{\alpha}+\xi_{0}\right), \\
& u_{8}=8 c k-12 c k \\
& \cdot \csc ^{2}\left(\frac{k x^{\alpha}}{\Gamma(1+\alpha)}+\frac{i}{\Gamma(1+\alpha)} \sqrt{\frac{k^{2}}{1+4 k^{2}}} t^{\alpha}+\xi_{0}\right) .
\end{aligned}
$$

\section{Conclusion}

In this paper, we used the extended Jacobi elliptic function expansion method for solving fractional differential equations and applied it to find exact solutions of the space-time fractional generalized reaction duffing equation, the space-time fractional bidirectional wave equations, and the space-time fractional symmetric regularized long wave (SRLW) equation. With the aid of Mathematica, we successfully obtained some new Jacobi elliptic function solutions including solitary wave solutions and trigonometric function solutions for these equations. This method is effective and can also be applied to other fractional differential equations.

\section{Conflict of Interests}

The author declares that there is no conflict of interests regarding the publication of this paper.

\section{Acknowledgments}

The author wishes to thank the referees for their valuable suggestions. He thanks Mr. Mehmet Ekici from the Department of Mathematics, Bozok University, Yozgat, Turkey. This paper is supported by the Scientific and Technological Research Council of Turkey (TUBITAK).

\section{References}

[1] K. S. Miller and B. Ross, An Introduction to the Fractional Calculus and Fractional Differential Equations, John Wiley \& Sons, New York, NY, USA, 1993.

[2] A. A. Kilbas, H. M. Srivastava, and J. J. Trujillo, Theory and Applications of Fractional Differential Equations, San Diego, Calif, USA, Elsevier, 2006.

[3] I. Podlubny, Fractional Differential Equations, vol. 198 of Mathematics in Science and Engineering, Academic Press, San Diego, Calif, USA, 1999.

[4] A. M. A. El-Sayed, S. Z. Rida, and A. A. M. Arafa, "Exact solutions of fractional-order biological population model," Communications in Theoretical Physics, vol. 52, no. 6, pp. 992996, 2009.

[5] M. Safari, D. D. Ganji, and M. Moslemi, "Application of He's variational iteration method and Adomian's decomposition method to the fractional KdV-Burgers-KURamoto equation," Computers \& Mathematics with Applications, vol. 58, no. 11-12, pp. 2091-2097, 2009.

[6] M. Inc, "The approximate and exact solutions of the spaceand time-fractional Burgers equations with initial conditions by variational iteration method," Journal of Mathematical Analysis and Applications, vol. 345, no. 1, pp. 476-484, 2008.

[7] G.-C. Wu and E. W. Lee, "Fractional variational iteration method and its application," Physics Letters A, vol. 374, no. 25, pp. 2506-2509, 2010.

[8] F. Fouladi, E. Hosseinzadeh, A. Barari, and G. Domairry, "Highly nonlinear temperature-dependent fin analysis by variational iteration method," Heat Transfer Research, vol. 41, no. 2, pp. 155-165, 2010.

[9] L. Song and H. Zhang, "Solving the fractional BBM-Burgers equation using the homotopy analysis method," Chaos, Solitons and Fractals, vol. 40, no. 4, pp. 1616-1622, 2009.

[10] S. Abbasbandy and A. Shirzadi, "Homotopy analysis method for multiple solutions of the fractional Sturm-Liouville problems," Numerical Algorithms, vol. 54, no. 4, pp. 521-532, 2010.

[11] H. Bararnia, G. Domairry, M. Gorji, and A. Rezania, "An approximation of the analytic solution of some nonlinear heat transfer in fin and 3D diffusion equations using HAM," Numerical Methods for Partial Differential Equations, vol. 26, no. 1, pp. 1-13, 2010.

[12] M. M. Rashidi, G. Domairry, A. DoostHosseini, and S. Dinarvand, "Explicit approximate solution of the coupled KdV equations by using the homotopy analysis method," International Journal of Mathematical Analysis, vol. 2, no. 9-12, pp. 581-589, 2008. 
[13] Z. Z. Ganji, D. D. Ganji, A. D. Ganji, and M. Rostamian, "Analytical solution of time-fractional Navier-Stokes equation in polar coordinate by homotopy perturbation method," Numerical Methods for Partial Differential Equations, vol. 26, no. 1, pp. 117-124, 2010.

[14] K. A. Gepreel, “The homotopy perturbation method applied to the nonlinear fractional Kolmogorov-Petrovskii-Piskunov equations," Applied Mathematics Letters, vol. 24, no. 8, pp. 14281434, 2011.

[15] P. K. Gupta and M. Singh, "Homotopy perturbation method for fractional Fornberg-Whitham equation," Computers \& Mathematics with Applications, vol. 61, no. 2, pp. 250-254, 2011.

[16] J. Guy, "Lagrange characteristic method for solving a class of nonlinear partial differential equations of fractional order," Applied Mathematics Letters, vol. 19, no. 9, pp. 873-880, 2006.

[17] M. Cui, "Compact finite difference method for the fractional diffusion equation," Journal of Computational Physics, vol. 228, no. 20, pp. 7792-7804, 2009.

[18] Q. Huang, G. Huang, and H. Zhan, "A finite element solution for the fractional advection-dispersion equation," Advances in Water Resources, vol. 31, no. 12, pp. 1578-1589, 2008.

[19] Z. Odibat and S. Momani, "Fractional Green function for linear time-fractional equations of fractional order," Applied Mathematics Letters, vol. 21, pp. 194-199, 2008.

[20] S. Zhang and H.-Q. Zhang, "Fractional sub-equation method and its applications to nonlinear fractional PDEs," Physics Letters A, vol. 375, no. 7, pp. 1069-1073, 2011.

[21] S. Guo, L. Mei, Y. Li, and Y. Sun, "The improved fractional sub-equation method and its applications to the space-time fractional differential equations in fluid mechanics," Physics Letters A, vol. 376, no. 4, pp. 407-411, 2012.

[22] B. Lu, "Bäcklund transformation of fractional Riccati equation and its applications to nonlinear fractional partial differential equations," Physics Letters A, vol. 376, no. 28-29, pp. 2045-2048, 2012.

[23] B. Tang, Y. He, L. Wei, and X. Zhang, "A generalized fractional sub-equation method for fractional differential equations with variable coefficients," Physics Letters A, vol. 376, no. 38-39, pp. 2588-2590, 2012.

[24] B. Zheng and C. Wen, "Exact solutions for fractional partial differential equations by a new fractional sub-equation method," Advances in Difference Equations, vol. 2013, article 199, 2013.

[25] B. Lu, "The first integral method for some time fractional differential equations," Journal of Mathematical Analysis and Applications, vol. 395, no. 2, pp. 684-693, 2012.

[26] B. Zheng, " $\left(G^{\prime} / G\right)$-expansion method for solving fractional partial differential equations in the theory of mathematical physics," Communications in Theoretical Physics, vol. 58, no. 5, pp. 623-630, 2012.

[27] K. A. Gepreel and S. Omran, "Exact solutions for nonlinear partial fractional differential equations," Chinese Physics B, vol. 21, no. 11, Article ID 110204, 2012.

[28] A. Bekir and Ö. Güner, "Exact solutions of nonlinear fractional differential equations by $\left(G^{\prime} / G\right)$-expansion method," Chinese Physics B, vol. 22, no. 11, Article ID 110202, 2013.

[29] B. Zheng, "Exact solutions for some fractional partial differential equations by the $\left(G^{\prime} / G\right)$ method," Mathematical Problems in Engineering, vol. 2013, Article ID 826369, 13 pages, 2013.

[30] W.-H. Su, X.-J. Yang, H. Jafari, and D. Baleanu, "Fractional complex transform method for wave equations on cantor sets within local fractional differential operator," Advances in Difference Equations, vol. 2013, article 97, 2013.
[31] A. J. M. Jawad, M. D. Petković, and A. Biswas, "Modified simple equation method for nonlinear evolution equations," Applied Mathematics and Computation, vol. 217, no. 2, pp. 869-877, 2010.

[32] E. M. E. Zayed, "A note on the modified simple equation method applied to Sharma-Tasso-Olver equation," Applied Mathematics and Computation, vol. 218, no. 7, pp. 3962-3964, 2011.

[33] E. M. E. Zayed and S. A. H. Ibrahim, "Exact solutions of nonlinear evolution equations in mathematical physics using the modified simple equation method," Chinese Physics Letters, vol. 29, no. 6, Article ID 060201, 4 pages, 2012.

[34] G. Jumarie, "Modified Riemann-Liouville derivative and fractional Taylor series of nondifferentiable functions further results," Computers \& Mathematics with Applications, vol. 51, no. 9-10, pp. 1367-1376, 2006.

[35] H. Zhang, "Extended Jacobi elliptic function expansion method and its applications," Communications in Nonlinear Science and Numerical Simulation, vol. 12, no. 5, pp. 627-635, 2007.

[36] M. A. Abdou and A. Elhanbaly, "Construction of periodic and solitary wave solutions by the extended Jacobi elliptic function expansion method," Communications in Nonlinear Science and Numerical Simulation, vol. 12, no. 7, pp. 1229-1241, 2007.

[37] A. H. Bhrawy, M. A. Abdelkawy, and A. Biswas, "Cnoidal and snoidal wave solutions to coupled nonlinear wave equations by the extended Jacobi's elliptic function method," Communications in Nonlinear Science and Numerical Simulation, vol. 18, no. 4, pp. 915-925, 2013.

[38] Z.-B. Li and J.-H. He, "Fractional complex transform for fractional differential equations," Mathematical \& Computational Applications, vol. 15, no. 5, pp. 970-973, 2010.

[39] M. Eslami, B. Fathi Vajargah, M. Mirzazadeh, and A. Biswas, "Application of first integral method to fractional partial differential equations," Indian Journal of Physics, vol. 88, no. 2, pp. 177-184, 2014.

[40] A. H. Arnous and M. Mirzazadeh, "Bäcklund transformation of fractional Riccati equation and its applications to the space-time FDEs," Mathematical Methods in the Applied Sciences, 2014.

[41] B. Lu, "Bäcklund transformation of fractional Riccati equation and infinite sequence solutions of nonlinear fractional PDEs," Abstract and Applied Analysis, vol. 2014, Article ID 572052, 6 pages, 2014.

[42] W. Li, H. Yang, and B. He, "Exact solutions of the spacetime fractional bidirectional wave equations using the $\left(G^{\prime} / G\right)$ expansion method," Journal of Applied Mathematics, vol. 2014, Article ID 153706, 9 pages, 2014.

[43] J. L. Bona and M. Chen, "A Boussinesq system for two-way propagation of nonlinear dispersive waves," Physica D. Nonlinear Phenomena, vol. 116, no. 1-2, pp. 191-224, 1998.

[44] F. Xu, "Application of Exp-function method to symmetric regularized long wave (SRLW) equation," Physics Letters A, vol. 372, no. 3, pp. 252-257, 2008.

[45] J. F. Alzaidy, "The fractional sub-equation method and exact analytical solutions for some nonlinear fractional PDEs," The American Journal of Mathematical Analysis, vol. 1, no. 1, pp. 1419, 2013. 




Advances in

Operations Research

mansans

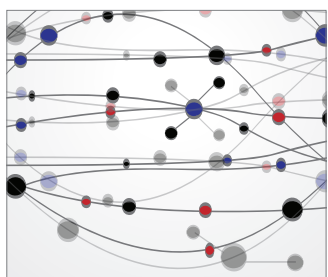

The Scientific World Journal
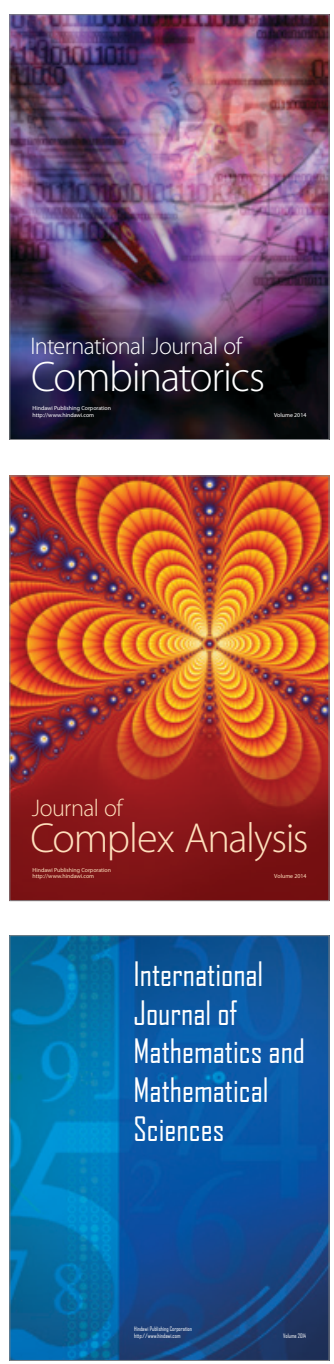
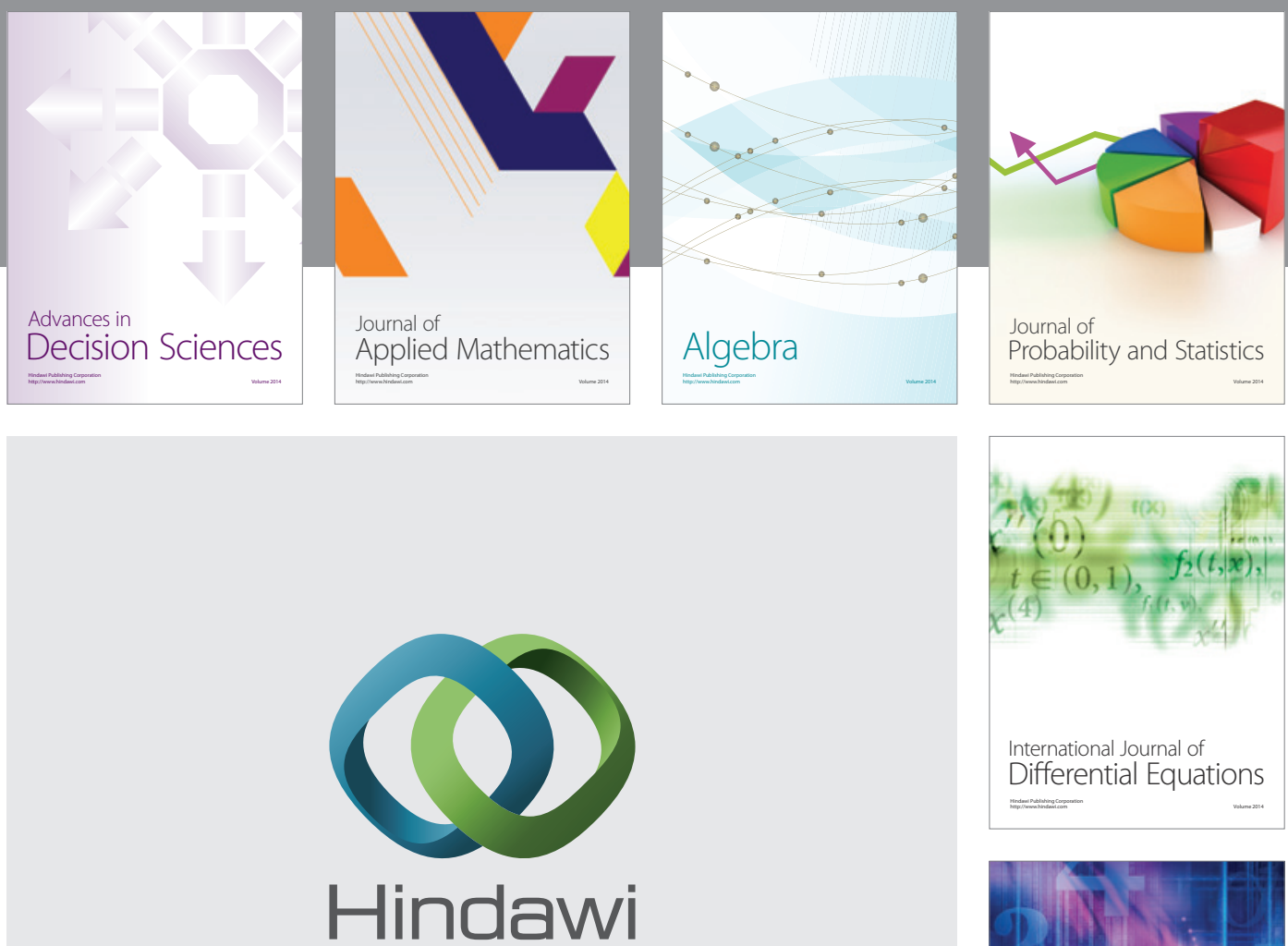

Submit your manuscripts at http://www.hindawi.com
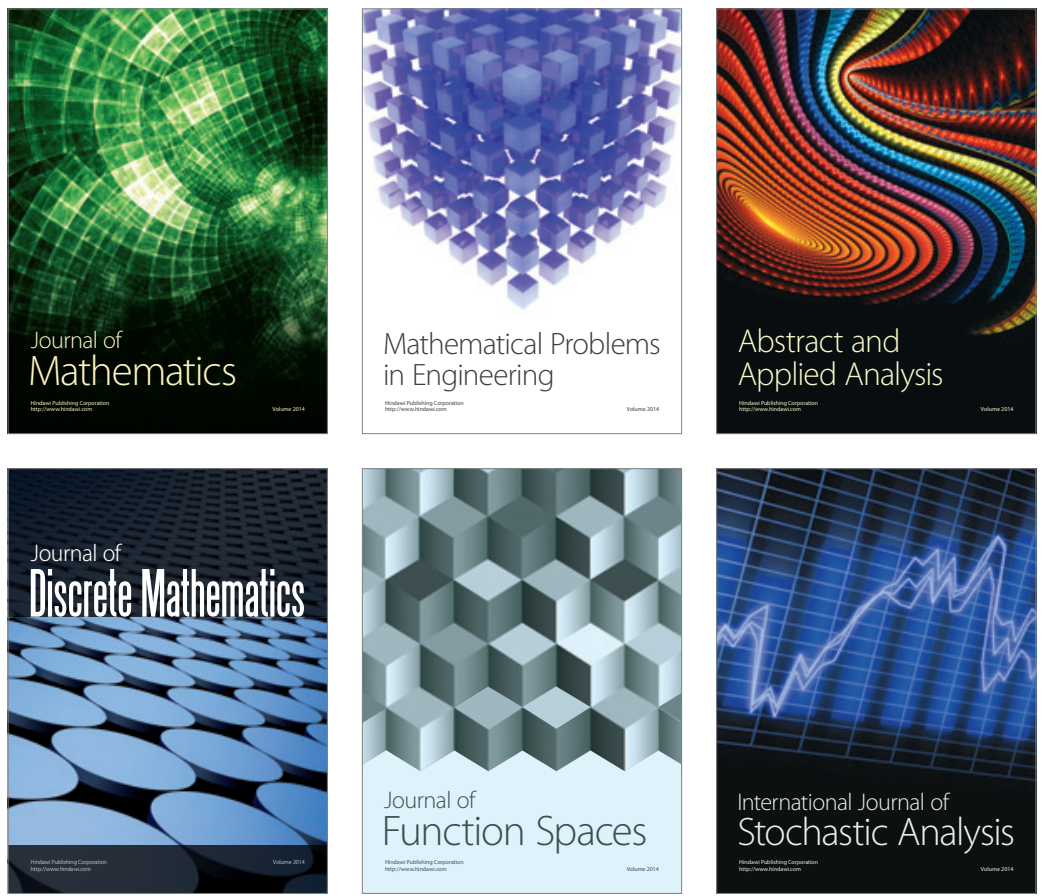

Journal of

Function Spaces

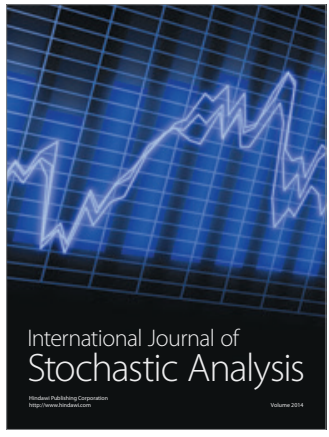

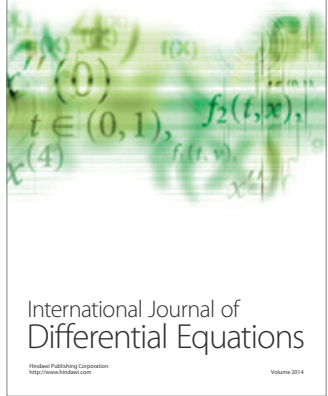
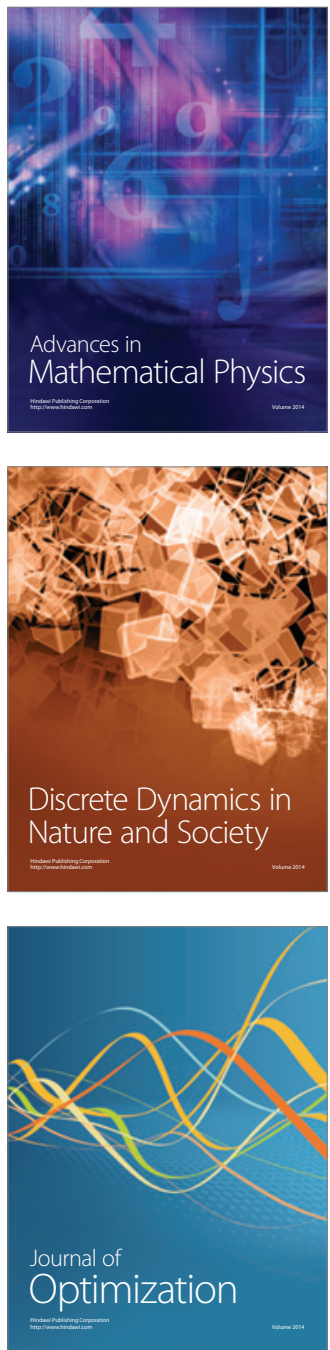